\title{
OSTEOPOROSIS TREATMENT IN PORTUGUESE PATIENTS WITH PSORIATIC ARTHRITIS - WHAT IS THE VALUE OF THE FRACTURE RISK ASSESSMENT TOOL (FRAX)?
}

Filipe Oliveira Pinheiro ${ }^{1, *}$, Maria Seabra Rato ${ }^{1}$, Bruno Miguel Fernandes ${ }^{1}$, Salomé Garcia ${ }^{1}$, Sara Santos ${ }^{1}$, Pedro Madureira ${ }^{1}$, Miguel Bernardes ${ }^{1,2}$, Lúcia Costa ${ }^{1}$

1. Centro Hospitalar e Universitário de São João, Porto, Portugal. 2. Faculdade de Medicina da Universidade do Porto, Porto, Portugal.

*Corresponding author: filipe.pinheiro92@gmail.com

\section{BACKGROUND}

Few studies have evaluated the prevalence and treatment of osteoporosis (OP) in patients with psoriatic arthritis (PsA), and many of these patients are not screened using dual energy -ray absorptiometry (DXA). FRAX makes it possible to stratify the risk and define which patients may benefit from anti-osteoporotic treatment, but its usefulness in this population is not well established. The aim of this study was to determine whether the application of FRAX changes the indication for anti-osteoporotic treatment, according to the Portuguese guidelines.

\section{MATERIALS AND METHODS}

In this cross-sectional study, we evaluated PsA patients from a tertiary hospital, registered in a national database (Reuma.pt), aged between 40 and 90 years and with a last consultation in 2019. Fracture risk assessment tool (FRAX) was applied in all of them, regardless of being under antiosteoporotic treatment and, when DXA was available, the femoral neck bone mineral density was used. Patients were stratified according to the risk of fracture, and those at high risk were considered candidates for antiosteoporotic treatment, according to national guidelines [FRAX $\geq 11 \%$ for major osteoporotic fracture (MOF) or $\geq 3 \%$ for hip fracture (HF) without DXA, or FRAX $\geq 9 \%$ for MOF or $\geq 2.5 \%$ for $\mathrm{HF}$, with DXA].

\section{RESULTS}

We included 100 patients, 52 females, with a mean age of $54.4 \pm 8.9$ years and a median disease duration of 10 (6-17) years. Seven patients were identified as having a high risk of fracture; applying femoral neck bone mineral density, 2 more patients with indication for treatment were recognized, totaling 9 patients. There was a low agreement between the indication for treatment based only on DXA and FRAX (Cohen's kappa $\mathrm{K}=0.066$ ). There was a moderate and significant correlation between percentage of risk of MOF by FRAX with and without DXA (Spearman's $\rho=0.804, p<0.001$ ); for the risk of HF by FRAX with and without DEXA, the correlation was weaker but still significant (Spearman's $p=0.439, p=0.004$ ). There was no association between the indication for treatment by FRAX and the performance of DXA (chi-square test, $p=0.597$ ), nor the fact of performing DXA significantly affected the risk of MOF (Wilcoxon test, $p=0.185$ ) or of HF (Wilcoxon test, $p=0.785$ ) by FRAX.

\section{CONCLUSIONS}

In line with Portuguese guidelines, FRAX seems to be, in itself, a very useful tool in patients with PsA, and the performance of DXA does not significantly alter the indication for antiosteoporotic treatment. 